\title{
Advanced feature selection for simplified pattern recognition within the damage identification framework
}

\author{
G. Manson* and R.J. Barthorpe \\ Dynamics Research Group, Department of Mechanical Engineering, University of Sheffield, Mappin Street, \\ Sheffield, S1 3JD, UK
}

\begin{abstract}
The paper is concerned with adopting a data-driven approach to damage detection and location on an aerospace structure without recourse to an artificial neural network. Five advanced features are selected, each detecting the removal of only one of five inspection panels on the structure. The features give perfect classification for damage location for single-site damage and $98.1 \%$ correct classification for multi-site damage scenarios, using a statistically calculated threshold. However, if the threshold values for two of the five features are altered slightly, $100 \%$ correct classification would be possible for single- and multi-site damage.
\end{abstract}

Keywords: Feature selection, damage location, structural health monitoring, neural networks

\section{Introduction}

There has been considerable success in recent years in considering the problem of damage identification as one of pattern recognition. The first author was involved in a body of research, funded by DERA/QinetiQ [1-4], on Structural Health Monitoring (SHM) which proposed a health monitoring system based upon novelty detection techniques. The aim of this overall body of research was to make as much progress as possible up the hierarchical fault detection structure [5],

Level 1. (DETECTION). The method gives a qualitative indication that damage might be present in the structure.

Level 2. (LOCALISATION). The method gives information about the probable position of the damage.

Level 3. (ASSESSMENT). The method gives an estimate of the extent of the damage.

Level 4. (PREDICTION). The method offers information about the safety of the structure, e.g. estimates a residual life.

whilst retaining, or even improving, the robustness of the lower level solutions.

The first two parts [1,2] of the earlier work were concerned with experimental validation of novelty detection techniques to carry out the first level of health monitoring on a simulated stiffened skin panel and also on the wing of a Gnat aircraft. Both these investigations met with a large degree of success.

The next two parts $[3,4]$ extended these techniques to levels 2 and 3 in the damage hierarchy, respectively. These were also conducted on the wing of the Gnat aircraft. Reference [3] was concerned with determining which of nine inspection panels had been removed by constructing a network of novelty detectors, one for each region of the structure, and using a neural network to interpret the patterns and suggest which panel had been removed. Reference [4] extended this work to damage assessment by using a neural network classifier, in conjunction with

\footnotetext{
*Corresponding author. E-mail: graeme.manson@sheffield.ac.uk.
} 


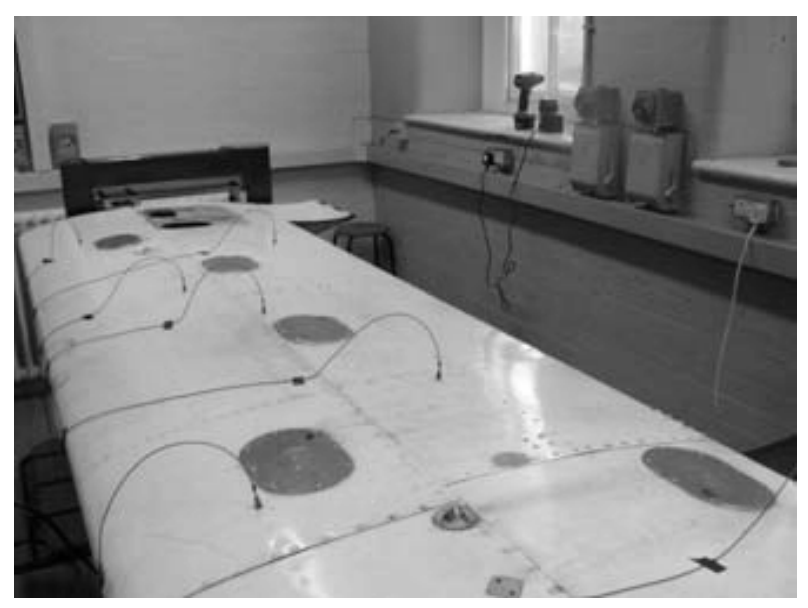

Fig. 1. Piper Tomahawk wing with five removable inspection panels.

regression neural networks, to, not only detect damage and locate which of three inspection panels were damaged, but also return a measure of the damage severity. Both studies gave very encouraging results; comparable with previous results concerning simulated data or simple laboratory structures.

Whilst the previous work undoubtedly made significant progress up the damage identification hierarchy, this progression was dependent upon the training of neural networks to provide a mapping between the novelty values and the damage location or severity. The training phase of the neural network requires large amounts of data from each possible damage scenario in order to optimise the network weights. In a realistic situation regarding monitoring of a high-cost structure, it will be infeasible to damage the structure at all, let alone damaging the structure to provide large amounts of data for all possible damage scenarios. The current work presents the first stage in the development of a new framework for the data-driven approach to structural health monitoring which, it is hoped, will eventually permit the field to make the transfer from lab to in-service structure. This first stage is concerned with removing the need for artificial neural networks for damage location by devoting greater effort to the feature selection process. The resulting advanced features will make the subsequent classification a straightforward process and, hopefully, address the issue of multiple damage occurrences in a sensible manner. It should be made very clear that the current work is still reliant upon the acquisition of exemplary data from the damaged structure. That said, if the first stage is successful, subsequent stages in the framework development will be to entirely remove the need for data from damaged structures and provide robustness against the effects of non-damaging variability.

The paper is laid at as follows: Section 2 introduces the structure under investigation, the data acquisition procedure and the test programme whilst Section 3 details the advanced feature selection process. Section 4 gives the results from the selected features for single and multiple damage occurrences before the paper is completed with some discussion.

\section{Test set-up and data capture}

\subsection{Test structure}

The structure under investigation in the current work was the starboard wing of Piper Tomahawk trainer aircraft as shown in Fig. 1. Acquisition of this wing permits the Dynamics Research Group at Sheffield to continue the programme of SHM work reported in [1-4].

As was the case with the previous wing, it was not desired that the wing actually be damaged and so it was decided that the removal of inspection panels would again be used to simulate damage scenarios in a reversible manner. On this occasion, five inspection panels were identified which would be systematically removed during the test programme. Figure 2 shows a schematic of the wing indicating the approximate locations of the panels and the accelerometers used to measure the vibration response of the wing. 

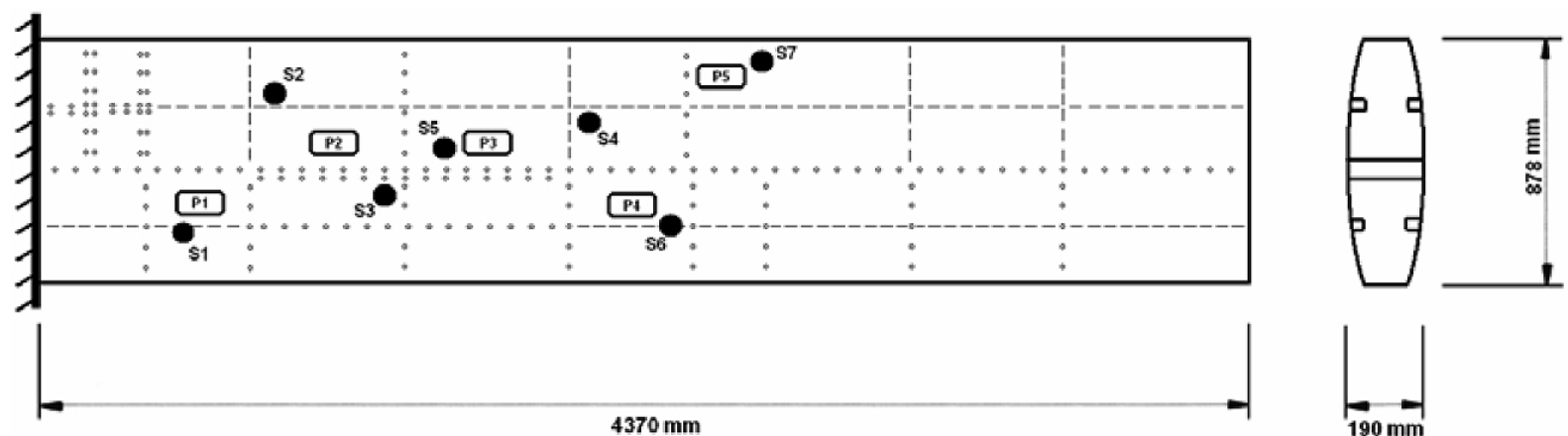

Fig. 2. Schematic of wing indicating locations of five inspection panels (P1-P5) and seven accelerometers (S1-S7).

The size of each panel was $200 \times 135 \mathrm{~mm}$ and each was attached to the wing via eight screws. In order to reduce one aspect of variability in the acquisition process, panels were attached using a torque-controlled screwdriver with the same torque setting throughout.

\subsection{Data acquisition}

Contrary to the previous studies, the base measurements chosen in this programme were frequency response functions (FRFs) as opposed to transmissibilities. There are three main reasons for this decision: (1) it is now the authors' view that SHM is more likely to be successful when the aircraft is grounded and therefore the input excitation can be controlled and measured, as opposed to relying on ambient excitation for in-flight monitoring, (2) it is now the authors' opinion that examination of FRFs with response points situated near the damage location is more likely to be successful than acquiring transmissibility data "across" the damage locations and (3) FRFs are a more familiar measurement in the dynamics community than transmissibilities. The veracity of the second statement will be tested within a subsequent study which will compare the use of transmissibility measurements against FRFs.

The wing was excited using a Gearing and Watson electrodynamic shaker attached to the underside of the wing on the central stiffener close to the location of Panel P2. The data acquisition process was controlled using LMS software running on a Dell PC connected to a DIFA Scadas acquisition system. A Gaussian white noise input excitation was generated within the LMS software and transmitted to the shaker via the DIFA Scadas and a Gearing and Watson power amplifier. Acceleration data from the seven PCB piezoelectric accelerometers on the top surface of the wing and force input data from the PCB force gauge attached to the shaker were recorded by the DIFA system and transmitted to the PC for processing within the LMS software. Figure 3 shows the acquisition set-up.

As stated above, the base measurements which were recorded were FRFs. The frequency range was $0 \mathrm{~Hz}$. to $2048 \mathrm{~Hz}$. with $0.5 \mathrm{~Hz}$. resolution giving a total of 4097 spectral lines in each FRF. Five averages were used which represented a compromise between acquisition time and noise reduction. It should be stated that the novelty detection procedure takes into account the measurement noise on the data to construct its models of normality. Figure 4 shows a typical FRF over the entire frequency range of interest and over a narrower frequency range. The test programme required FRFs to be acquired from a number of test configurations which will be explained below. In order to use novelty detection techniques within the feature selection techniques, a total of 100 FRFs were recorded from each of the seven accelerometers during each test configuration.

\subsection{Test configurations}

There were two main objectives of the current work. The first objective was to investigate whether there existed, for each of the five inspection panels, a distinguishing feature, derived from the acquired data, which was sensitive to the removal of only that panel and insensitive to the removal of the other four panels. The second objective was contingent on the first being successful and was concerned with examining whether these distinguishing features (selected using data from the undamaged structure and from the structure with one panel removed) behaved in the desired manner when presented with data acquired when more than one panel was removed from the wing. 


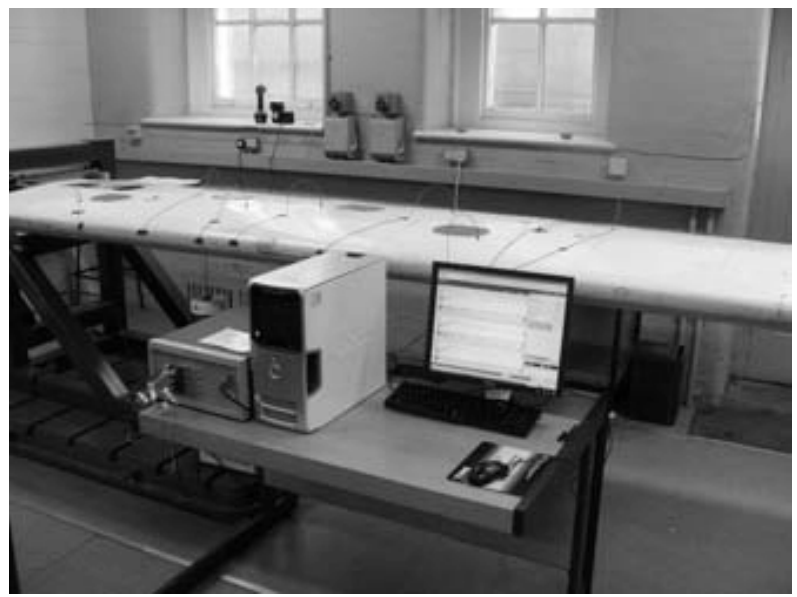

Fig. 3. Data acquisition set-up.
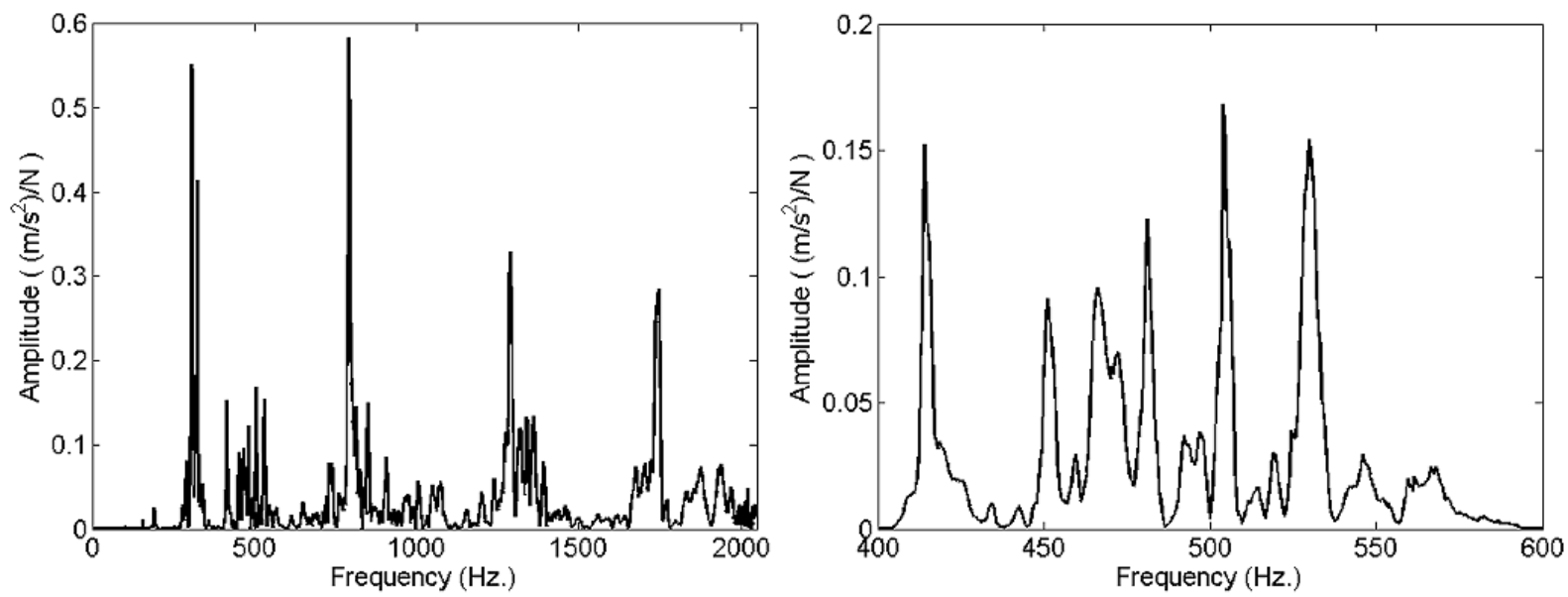

Fig. 4. Undamaged structure FRF from sensor 1. (a) 0-2048 Hz. (b) 400-600 Hz.

A large test programme was constructed to investigate the feasibility of the above objectives. In order to capture the variability caused by removing and re-attaching the inspection panels, a total of 22 normal condition configurations were conducted on the undamaged structure throughout the test programme. For five inspection panels there are 31 possible combinations of single and multiple panel removals. Again, for the purposes of being robust against variability caused by panel attachment, two configurations were conducted for each of these 31 combinations from the damaged structure.

The order of the test configurations was as follows: N1, D1, N2, D2, N3, D3, N4, D4, N5, D5, N6, D1 R2, N7, D2_R2, N8, D3_R2, N9, D4_R2, N10, D5_R2, N11, D1 2, D1 3, D1 4, D1 5, D2 3, N12, D2 4, D2 5, D3 4,

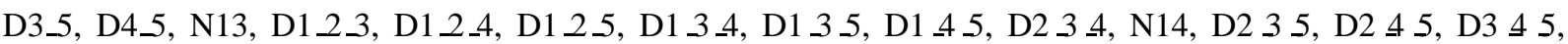
N15, D1_2_3_4, D1_2_3 5, D1 2_4_5, D1 3 4 _5, D2 3 4 ـ5, N16, D1 23 4 5, N17, D1 2 R2, D1 3 R2, D1 4 R2, D1_5_R2, D2_3_2, N18, D2_4_2, D2 5ـR2, D3_4R2, D3 5 R2, D4 5 R2, N19, D1 23 R2, D1 24 R2, D1_2_5_R2, D1_3_4_R2, D1_3 5 R2, D2_3_4R2, D1_4 5 R2, N20, D2 3 5 R2, D2 45 R2, D3 45 R2, N21, D1_2_3_4_R2, D1_23_5_R2, D1 2_4_5_R2, D1_3_45R2, D2_3_4 5 R2, N22, D1 2345 R2 where N $x$ refers to the $x^{\text {th }}$ normal condition configuration from the undamaged structure and $\mathrm{D} x y z$ refers to the damage condition configuration with inspection panels $\mathrm{P} x, \mathrm{P} y$ and $\mathrm{P} z$ removed. $\mathrm{R} 2$ refers to the second repetition of the damage configuration. Unfortunately, during the data acquisition, after test configuration N13 and before D1 23 , sensor 
2 was accidentally dislodged. This will need to be considered later in the advanced feature selection phase of the work.

\subsection{Data organisation}

The test programme meant that, for each accelerometer, a total of 2200 normal condition FRFs and 6200 damage condition FRFs were recorded. Upon completion of the test programme, the data were stored in two large matrices, in preparation for data processing within MATLAB. The normal condition matrix was of size $7 \times 4097 \times 2200$ representing the seven accelerometers, 4097 spectral lines in each FRF and 22 normal configurations, each of 100 FRFs. Similarly, the damage condition matrix was of size $7 \times 4097 \times 6200$ representing the seven accelerometers, 4097 spectral lines in each FRF and 2 repetitions of 31configurations, each of 100 FRFs. In the present study, only the FRF magnitude information will be used for identification of features. At a later date, other aspects of the acquired data, such as phase information, will be investigated regarding their suitability for advanced feature selection.

\section{Advanced feature selection process}

As stated in the last section, the first objective of this study was to investigate whether there existed distinguishing features which were sensitive to the removal of only one panel and insensitive to the removal of the other four panels. In this section, the process for identifying these features will be described.

\subsection{Novelty detection using a univariate outlier test}

In order to try to find out if any of the spectral lines of any of the FRFs may be considered as advanced features, it was decided that a univariate outlier test be performed for each spectral line for each sensor. The normal data set was divided into a training set and a testing set, each of which contained 1100 of the 2200 normal condition test observations. The normal training set, which included test configurations N1, N3, N5, .., N21, was used to construct the novelty detectors for each spectral line, against which all the normal testing set and damage set observations could be tested. The purpose of the normal testing set, which included test configurations N2, N4, $\mathrm{N} 6, \ldots, \mathrm{N} 22$, was to check that the chosen features were capable of not only detecting the presence of a particular damage but also of correctly classifying normal condition data as such.

The process for constructing the novelty detectors was as follows. First, the mean and standard deviations for the magnitude of each spectral line of each FRF from the 1100 normal training observations were calculated. These training mean and standard deviation values were used to calculate univariate measures of discordancy for the magnitude of every spectral line from each FRF for the 1100 normal testing observations and 6200 damage observations using the formula given in Eq. (1) where $\zeta$ is the discordancy measure for some spectral line magnitude $x$ in the test data set when compared to the mean, $\mu$, and standard deviation, $\sigma$, of the 1100 normal training data tests for that particular FRF.

$$
\zeta=\frac{|x-\mu|}{\sigma}
$$

More details concerning outlier analysis may be found in the standard reference [6].

It should be stated here that the authors are certainly not proposing that the best feature for damage detection and location is going to be the magnitude of a single spectral line of an FRF. It was found, not surprisingly, that the spectral lines around the chosen line also behave in a very similar manner and it is likely that a much more robust feature would be formed by considering spectral ranges. For the present study, the single spectral line feature suffices to illustrate the potential for a more complex feature. 
Table 1

Best single spectral line advanced features

\begin{tabular}{cccc}
\hline Panel removal & Sensor number & Spectral line & Measure value \\
\hline P1 & 1 & 495 & 29.9 \\
P2 & 2 & 333 & 7.46 \\
P3 & 5 & 396 & 16.1 \\
P4 & 6 & 257 & 12.8 \\
P5 & 7 & 186 & 28.7 \\
\hline
\end{tabular}

\subsection{Advanced feature selection measures}

With respect to discordancy measures, the characteristics of the advanced features whose identification is desired, is that it will result in high discordancy values when presented with data from the structure with its particular panel removed and low discordancy values when presented with data from the structure with its particular panel attached (regardless of whether other panels have been removed). In order to classify the suitability of all of these potential features, an objective measure is required. There are many possibilities for suitable measures but an example of the one used in the current work is given below in Eq. (2). This measure is designed to identify features which are able to detect the removal of panel P1, hence the Subscript 1 in $M_{1}$. This measure is simply a ratio of the sum of all 200 discordancy values from test configurations D1 and D1 R2 to the 800 discordancy values from test configurations D2, D2_R2, D3, D3_R2, D4, D4_R2, D5 and D5_R2.

$$
M_{1}=\frac{\sum \zeta_{1}}{\sum \zeta_{2}+\sum \zeta_{3}+\sum \zeta_{4}+\sum \zeta_{5}}
$$

In a similar fashion $M_{2}, M_{3}, M_{4}$ and $M_{5}$ measures were also calculated for each of the 4097 spectral lines from each of the seven FRFs.

It should be noted that these measures are only calculated using the damage data where single inspection panels have been removed. Once the feasibility of the process of identifying these advanced features is completed, the data acquired from the structure with multiple panel removals will be presented to the features to see how they behave.

\section{Results}

In this section, the process which was outlined in the previous section will be applied to the data acquired from the Tomahawk wing during the test programme to try to identify advanced features which are only sensitive to damage at one location.

\subsection{Advanced feature selection measures results}

The procedure outlined in Subsection 3.2 was followed and measures were obtained as per Eq. (2) for each of the 4097 spectral lines from the seven FRFs to try to find the best spectral line for detecting the removal of panel P1 whilst not detecting the removal of the other four panels. This process was repeated to find the best spectral lines to detect the removal of panels $\mathrm{P} 2, \mathrm{P} 3, \mathrm{P} 4$ and $\mathrm{P} 5$. The results of this process are given in Table 1 .

It was stated earlier that sensor 2 was dislodged during the data acquisition programme and so, for the multiple damage analysis, it is also worth identifying the best feature for detecting the removal of panel P2 which was not acquired from sensor 2. This feature was spectral line 876 from sensor number 3 which had a measure of 5.00 (compared with 7.46 for the best sensor 2 measure). In the following subsection the success of the identified features will be assessed.

Before advancing to this analysis it should be pointed out that, for all five damage scenarios, the best sensor was one which was close to the damage. One should not be over-surprised by this finding but it may raise concerns regarding the need for knowledge of potential damage locations when considering sensor placement. This will be addressed after the chosen features are analysed in the next three subsections. 


\subsection{Advanced features results for single site damage}

In order that a test measurement can be declared as an outlier, a threshold measurement is first required. In this work, the threshold was calculated based upon a Monte-Carlo simulation of 100000 trials using extreme value statistics [7] for a univariate problem with 1100 observations. The $99.99 \%$ exclusive threshold was calculated to be 5.42. If a discordancy value is greater than this threshold it will be inferred that a particular panel is removed and below the threshold it will be inferred that the panel is in situ. Although this threshold value will be used throughout, the actual value is not of primary concern but rather that there exists some threshold, for each of the advanced features, which is capable of identifying when the panel of interest has been removed.

The success of the selected features is shown in Fig. 5. It can be seen in plot (a) that feature 1 manages to classify all 200 of the D1 observations (removal of Panel P1) as outliers due to being above the 5.42 threshold value (shown as a dashed horizontal red line) and all 800 of the other single site damage (removal of P2, P3, P4 and P5) observations as being below threshold. Plots (b) to (e) show exactly the same behaviour with features 2 to 5 also being sensitive only to the removal of the panel for which they were chosen. Plot (f) in Fig. 5 shows the results of the standby feature 2 (ie. not using sensor 2 data) and it may be seen that it also gives $100 \%$ correct classification for the single site damage. The behaviour of these features when presented with data from multiple damage scenarios will be investigated shortly. Before that, the behaviour of these advanced features when presented with the normal testing data will be presented in the next subsection.

At the end of the previous subsection, it was noted that the chosen features were always from a nearby sensor. This does not necessarily mean that one requires a sensor to be located near to the damage location. It is rather indicating that, if one is interested in obtaining a feature which is only sensitive to damage at a particular location, then it is likely that this feature will be extracted from data acquired from one of the sensors closest to the damage. There is a subtle difference between the previous two sentences.

Further examination of the feature selection process discussed in Subsection 4.1 reveals that there exist advanced features, drawn from sensors other than those given in Table 1, that are capable of given perfect single site classification. For the detection of panel P2 removal, there are suitable features drawn from sensors 2, 3, 4, 5, 6 and 7. For panel P3 removal, there are suitable features drawn from sensors 2, 3, 4 and 5. The situation is less impressive for the other panel removals. For panel P1 removal, in addition to the many features from sensor 1, there exist a few features from sensor 3 which are just capable of perfect single site classification. Similarly for panel P4 removal, in addition to the many features from sensor 6, there exist a few features from sensor 1 which are just capable of perfect single site classification. For panel P5 removal, however, the only features which are capable of perfect single site classification are from sensor 7.

It must be reiterated that it is authors' opinion that this does not indicate that one must have a priori information regarding the potential damage locations, but rather that this approach will be likely to draw the advanced features from one of the sensors nearest to the damage location. At the end of Subsection 4.4, these other features will be revisited to assess their ability to identify the multiple damage scenarios.

\subsection{Advanced features results for normal test data}

Whilst the priority of this work is to select advanced features which are sensitive only to damage at one location, it is also necessary to ensure that the chosen features are also able to correctly classify test data which has come from the undamaged structure. For this purpose a normal testing set, independent of the normal training set, was constructed using the test configurations N2, N4, N6, .., N22. The word independent here only means that the even number test configurations did not contribute to forming the training set statistics. In order to demonstrate true reproducibility, it would be preferable to record the normal test data at a significant period after the original training set had been recorded. For each of the advanced features, the discordancy values from the relevant 1100 normal test observations were compared to the threshold value to check that there were no excursions over the threshold, which would represent a false positive result. Due to space limitations, the results will be summarised rather than displayed graphically.

It transpired that for all five advanced features, detailed in Table 1, all 1100 of the normal test observations were under the 5.42 threshold. The maximum normal test discordancy values which were observed were 2.30, 2.86, 

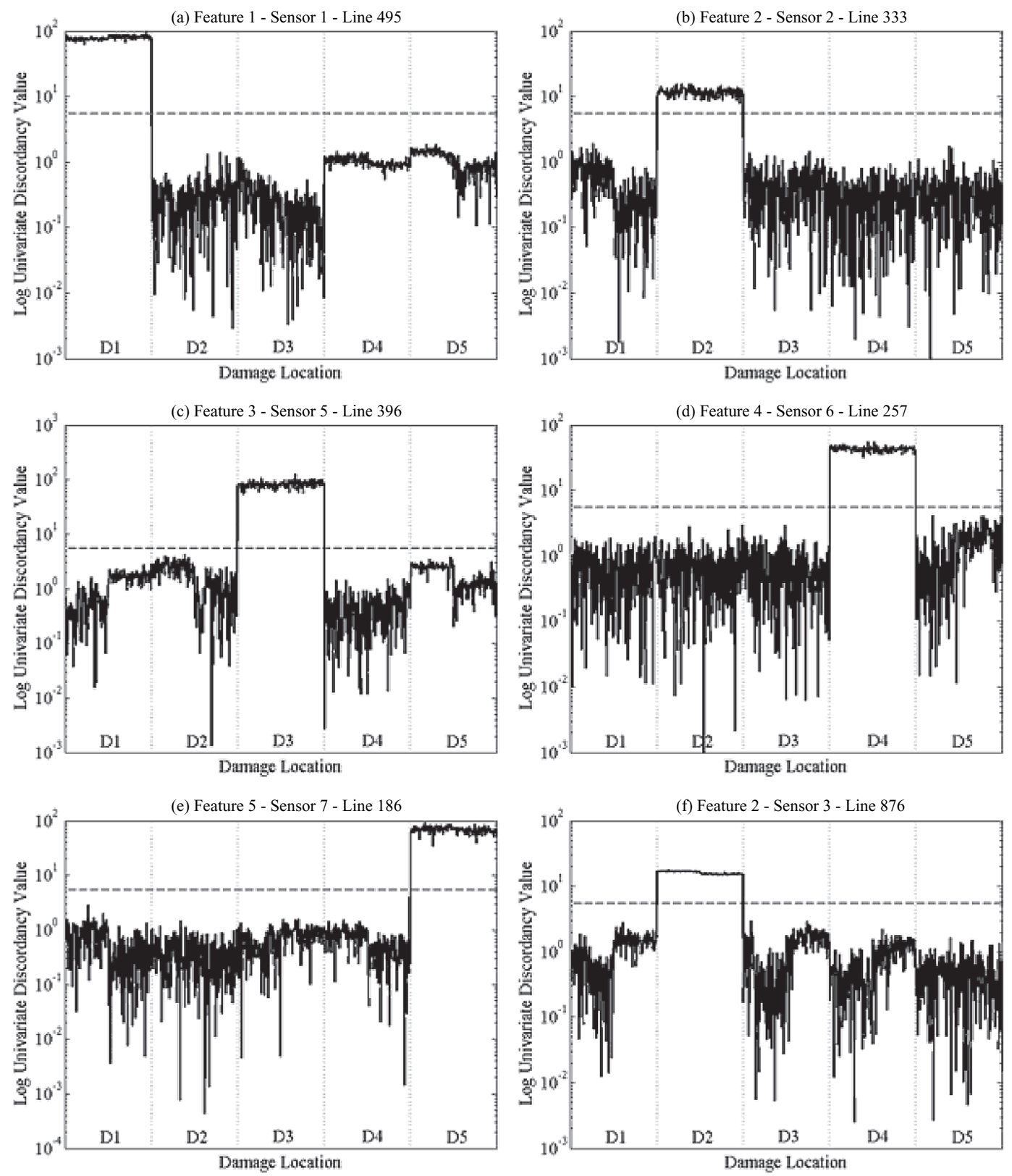

Fig. 5. Univariate outlier results for selected advanced features when presented with single site damage.

3.90, 4.75 and 2.80 for feature 1, 2, 3, 4 and 5 respectively. The only slight problem which arose concerned the standby feature 2 (ie. not using sensor 2 data but rather spectral line 876 from sensor 3 ) which resulted in 1 out of the 1100 normal test observation returning a discordancy value of 5.46, very slightly above the 5.42 threshold. As stated earlier, the actual threshold value is not of primary concern, but the behaviour of the advanced features when presented with the normal testing data is very encouraging. In the next subsection, the performance of the features when presented with the multiple site damage data will be reported.

\subsection{Advanced features results for multiple site damage}

As encouraging as the results are for advanced feature performance when presented with data from single site da- 
Table 2

Summary of univariate outlier results for advanced features when presented with multiple site damage

\begin{tabular}{|c|c|c|c|c|c|c|}
\hline $\begin{array}{l}\text { Damage } \\
\text { Scenario }\end{array}$ & $\begin{array}{c}\text { Feature } 1> \\
\text { threshold }\end{array}$ & $\begin{array}{c}\text { Feature } 2> \\
\text { threshold }\end{array}$ & $\begin{array}{c}\text { Feature } 3> \\
\text { threshold }\end{array}$ & $\begin{array}{c}\text { Feature } 4> \\
\text { threshold }\end{array}$ & $\begin{array}{c}\text { Feature } 5> \\
\text { threshold }\end{array}$ & $\begin{array}{c}\text { Feature } 2 \mathrm{~S}> \\
\text { threshold }\end{array}$ \\
\hline D1_2 & 200 & 200 & 1 & 0 & 0 & 200 \\
\hline D1_3 & 200 & 0 & 200 & 1 & 0 & 0 \\
\hline D1_4 & 200 & 0 & 0 & 200 & 0 & 0 \\
\hline D1_5 & 200 & 0 & 0 & 1 & 200 & 0 \\
\hline D2_3 & 0 & 200 & 200 & 0 & 0 & 200 \\
\hline D2_4 & 0 & 200 & 0 & 200 & 0 & 200 \\
\hline D2_5 & 0 & 200 & 0 & 0 & 200 & 200 \\
\hline D3_4 & 0 & 0 & 200 & 200 & 0 & 0 \\
\hline D3_5 & 0 & 0 & 200 & 0 & 200 & 0 \\
\hline D4_5 & 0 & 0 & 0 & 200 & 200 & 0 \\
\hline D1_2_3 & 200 & 200 & 200 & 0 & 0 & 200 \\
\hline D1_2_4 & 200 & 200 & 7 & 200 & 0 & 200 \\
\hline D1_2_5 & 200 & 200 & 0 & 0 & 200 & 200 \\
\hline D1_3_4 & 200 & 0 & 200 & 200 & 0 & 0 \\
\hline D1_3_5 & 200 & 0 & 200 & 0 & 200 & 0 \\
\hline D1_4_5 & 200 & 0 & 86 & 200 & 200 & 0 \\
\hline D2_3_4 & 0 & 199 & 200 & 200 & 0 & 200 \\
\hline D2_3_5 & 0 & 200 & 200 & 0 & 200 & 200 \\
\hline D2_4_5 & 0 & 200 & 1 & 200 & 200 & 200 \\
\hline D3_4_5 & 0 & 0 & 200 & 200 & 200 & 0 \\
\hline D1_2_3_4 & 200 & 200 & 200 & 200 & 0 & 200 \\
\hline D1_2_3_5 & 200 & 199 & 200 & 0 & 200 & 200 \\
\hline D1_2_4_5 & 200 & 200 & 0 & 200 & 200 & 200 \\
\hline D1_3_4_5 & 200 & 0 & 200 & 200 & 200 & 0 \\
\hline D2_3_4_5 & 0 & 200 & 200 & 200 & 200 & 200 \\
\hline D1_2_3_4_5 & 200 & 198 & 200 & 200 & 200 & 200 \\
\hline
\end{tabular}

mage (Subsection 4.2), to some extent they are to be expected. Once it was ascertained that features existed that were capable of sensitivity to only one damage location, the results of Fig. 5 follow, to a certain extent, as the measures (such as given in Eq. (2) were designed to maximize the difference between the single site damage discordancy values.

What is unpredictable, is how these advanced features will behave when presented with data from the structure with multiple panels removed. The desired behaviour would be that, even with multiple panel removals, feature 1 will return a discordancy value over threshold whenever panel P1 is removed and below threshold when it is attached. Similar behaviour is desired from features 2 to 5 with regard to the status of panels P2 to P5.

As with the normal testing results, due to space limitations, the behaviour of the advanced features when presented with multiple damage data will be summarised. Table 2 shows how each of the five features and the standby feature 2 (labeled Feature 2S) behave for each of the multiple damage scenarios. Perfect classification for each damage scenario would consist of feature 1 detecting all 200 of the observations over threshold had panel P1 been removed and returning a 0 , if panel $\mathrm{P} 1$ had not been removed.

The results are incredibly encouraging with features1, 5 and $2 \mathrm{~S}$ returning perfect classification for all 26 of the multiple damage scenarios. Rather surprisingly, feature 2 performs well considering sensor 2 was re-attached during the test programme. Feature 2 returns false negatives for four of the 5200 observations. The discordancy values for these four cases are between 4.79 and 5.37 and therefore only slightly below the threshold value of 5.42. Feature 4 returns two false positives out of the 5200 observations with discordancy values of 5.81 and 5.75 , slightly over the threshold. In fact, for Feature 4, had the threshold been any value between 5.81 and 19.4, this feature would also have returned perfect classification.

The feature which has underperformed relative to the others is Feature 3. This feature returned a total of 95 false positives from the 5200 observations which still represents $98.1 \%$ correct classification for the multiple damage scenarios. The highest discordancy value of these 95 false positives was 7.05. However, as the true positive discordancy values are high, having a threshold value of between 7.05 and 31.4 would result in Feature 3 also returning perfect classification. 
Overall, if Feature2S were used in conjunction with Features 1, 3, 4 and 5, without changing the threshold value from 5.42, 5103 of the 5200 multiple damage observations would be completely correctly classified $(98.1 \%$ correct classification). If the threshold value for Feature 4 were raised very slightly and that for Feature 3 were raised a little more, all 5200 of the multiple damage observations could be completely correctly classified by all five features. This result is extremely encouraging.

At the end of Subsection 4.2, the relationship between damage location and sensor location was broached. It was argued that, although the best feature to diagnose damage at a particular location was always drawn from a nearby sensor, this should not be interpreted as the need for a priori knowledge of the damage location. It was shown that features drawn from other sensors were capable of independent damage location for single site damage. These same features were also assessed to see if any of them were able to perform multiple site damage location.

It transpired that for panel P2 removal, only the features drawn from sensors 2 and 3 were capable of multiple site location. For panel P3 removal, sensors 4 and 5 were able to provide features which gave perfect multiple site classification. For panel P1 removal, the best sensor 3 feature was capable of giving a very encouraging, although not quite perfect, multiple site classification and sensor 1 gave a perfect classification for the removal of panel P4. Overall, it is felt that this has gone some way to showing that it is not necessary to have a sensor very close to the location of the damage in order for the technique to work. This will be further addressed in a subsequent study using a greater number of sensors.

\section{Discussion and conclusions}

The results show that, for the structure under test, there exist distinguishing features which are sensitive to damage at one location yet insensitive to damage at all other locations. This essentially means that the first and second levels of Rytter's damage identification hierarchy, namely damage detection and location, can be addressed together without recourse to a neural network classifier. Neural network classifiers have two main problems when applied to damage identification. The first problem is the requirement for a large number of data from each damage scenario in order to train the network. Whilst the current work made use of data from the damage structure, these would not be required in the same quantity as for neural network training which, for generalisation purposes according to Tarassenko's guidelines for neural network usage [8], require around 10 observations per network weight. This would have resulted in 1700 single-site damage data observations for a neural network with 5 input nodes, 15 hidden nodes and 5 output nodes. This is more than the 1000 single-site observations used in the presented approach; a figure which could easily have been reduced considerably without any detrimental effect upon the results.

The second problem relates to the inability of a 1 of $M$ classifier to classify in a useful manner when presented with data from a scenario where multiple damage occurrences are present. The current work appears to have resulted in a set of features which generally behaves in the desired manner when presented with multiple damage data. There is a small issue regarding the setting of thresholds which needs to be addressed.

Whilst this initial phase of the work has undoubtedly been successful, there is large body of work which needs to be conducted before this technique will be mature enough to make the transition from lab to operating structure. The most important issue, as is often the case in the data-driven approach to SHM, is how the technique can be conducted without damaging the structure. In the current work, the advanced features were selected using normal condition data and single-site damage data whereas, in reality, one will not be permitted to damage a high-cost structure. The next phase of the work will address this issue on two fronts. The first approach will investigate the physical reason behind the successful features and assess whether they could be identified purely from the physics of the undamaged structure. The second approach will investigate whether these advanced features could be identified by inducing local non-damaging alterations to the structure, initially in the form of mass addition.

Two other issues which will have to be addressed are ensuring that the method can be made robust against non-damaging variability, such as environmental variations, and investigating other types of structure to see if they behave similarly.

\section{Acknowledgements}

The authors would like to thank Mr A. Williams for his help during the data acquisition phase of this work, Mr. F. Holz for his schematic drawing of the wing and Prof. K. Worden for valuable discussions. 


\section{References}

[1] K. Worden, G. Manson and D.J. Allman, Experimental Validation of a Structural Health Monitoring Methodology: Part I. Novelty Detection on a Laboratory Structure, Journal of Sound and Vibration 259 (2003), 323-343.

[2] G. Manson, K. Worden and D.J. Allman, Experimental Validation of a Structural Health Monitoring Methodology: Part II. Novelty Detection on a Gnat Aircraft, Journal of Sound and Vibration 259 (2003), 345-363.

[3] G. Manson, K. Worden and D.J. Allman, Experimental Validation of a Structural Health Monitoring Methodology: Part III. Damage Location on an Aircraft Wing, Journal of Sound and Vibration 259 (2003), 365-385.

[4] G. Manson, K. Worden and D.J. Allman, Experimental Validation of a Damage Severity Method, Proceedings of the 1st European Workshop on Structural Health Monitoring, Paris, France, 2002, pp. 845-852.

[5] A. Rytter, Vibration Based Inspection of Civil Engineering Structures, Ph.D. Thesis, University of Aalborg, Denmark, 1993.

[6] V. Barnett and T. Lewis, Outliers in Statistical Data, John Wiley and Sons, Chichester, 1994.

[7] E. Castillo, Extreme Value Theory in Engineering, Academic Press, 1988.

[8] L. Tarassenko, A Guide to Neural Computing Applications, Arnold, 1998. 

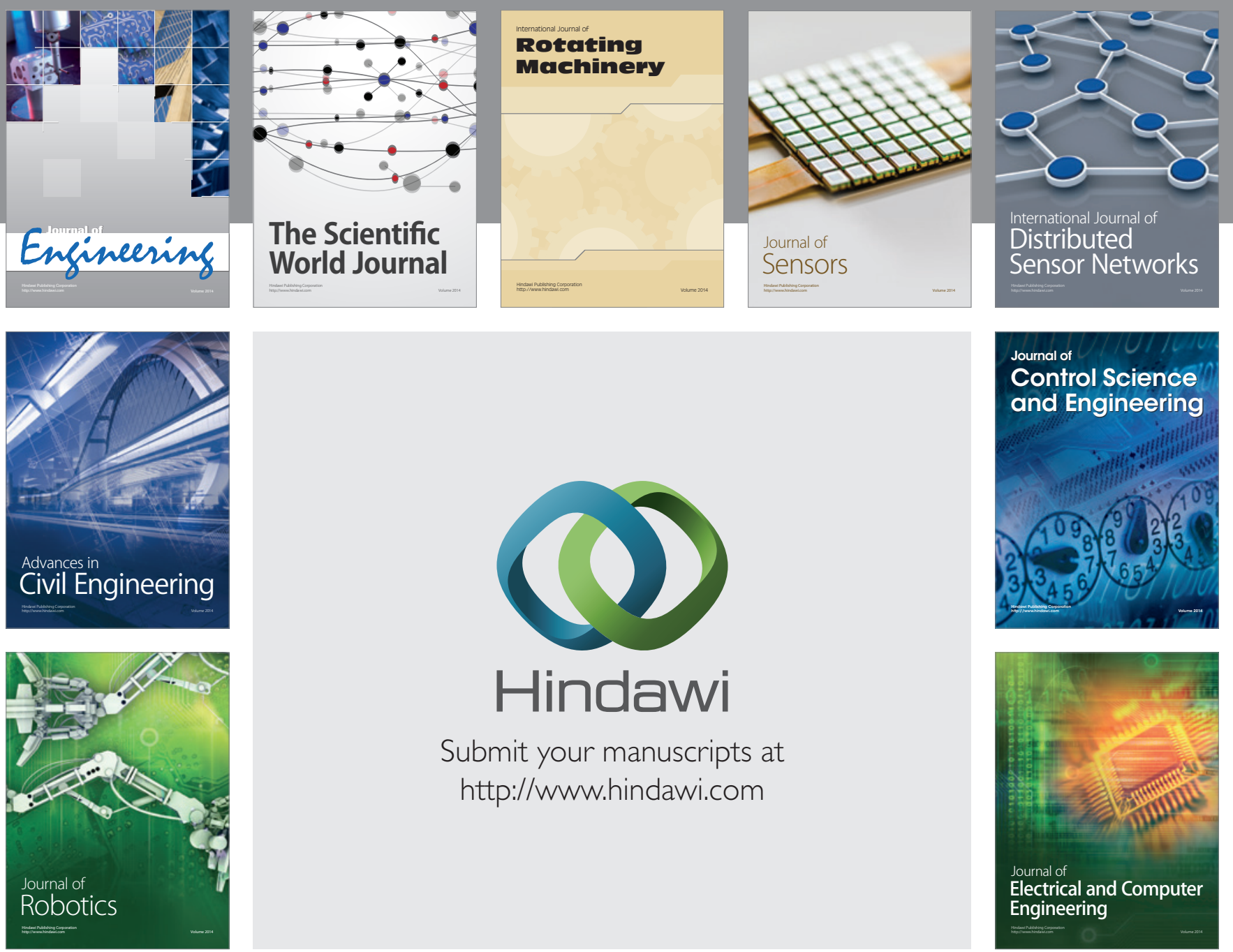

Submit your manuscripts at

http://www.hindawi.com
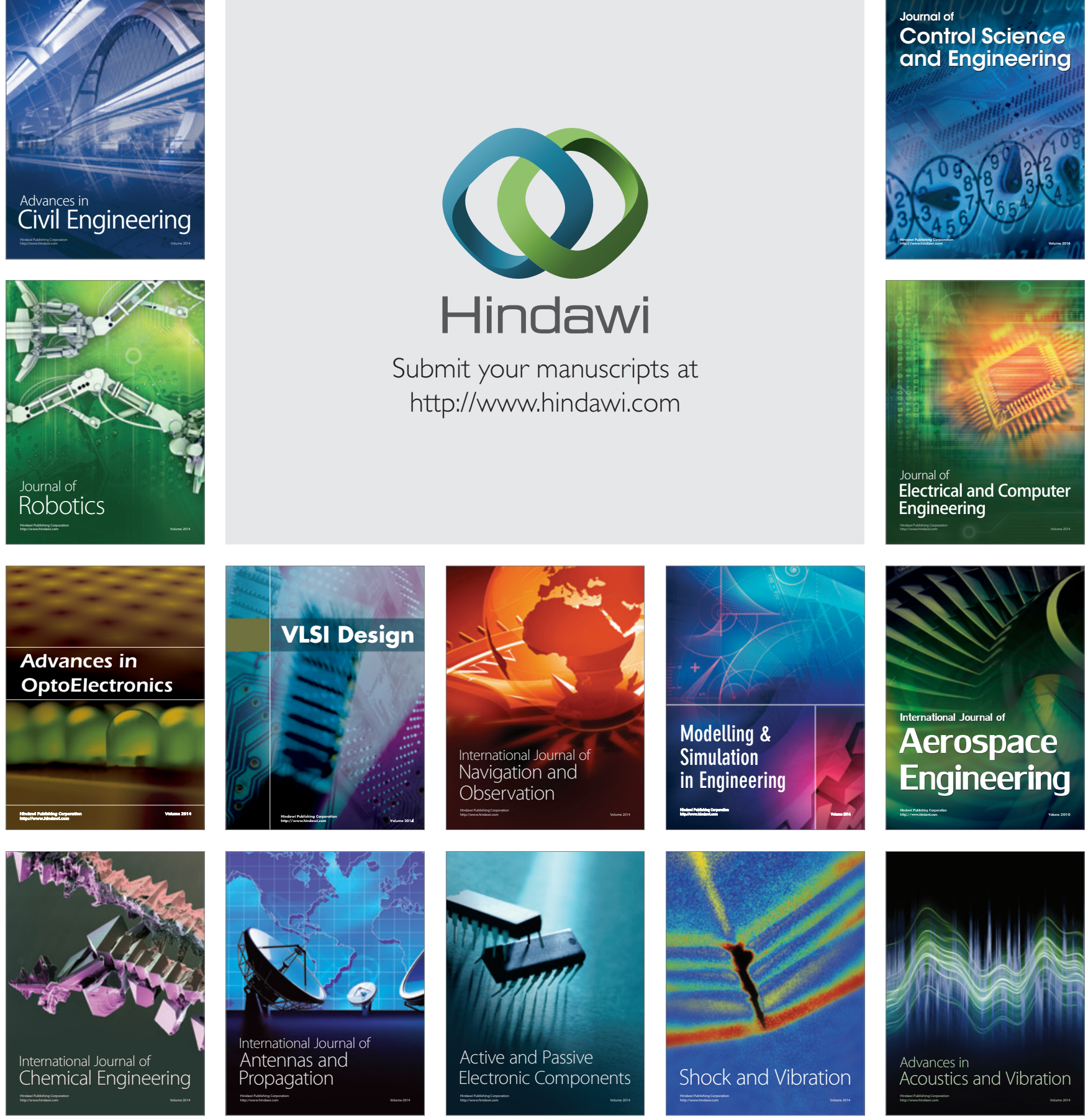